\title{
Local wavelet correlation: application to timing analysis of multi-satellite CLUSTER data
}

\author{
J. Soucek ${ }^{1,2}$, T. Dudok de Wit ${ }^{2}$, M. Dunlop ${ }^{3}$, and P. Décréau ${ }^{2}$ \\ ${ }^{1}$ Institute of Atmospheric Physics, Prague, Czech Republic \\ ${ }^{2}$ LPCE, CNRS and University of Orléans, 3A, avenue de la Recherche Scientifique, F-45071 Orléans cedex 2, France \\ ${ }^{3}$ Space Science and Technology Department, CCLRC Rutherford Appleton Laboratory, Chilton, Oxfordshire OX11 0QX, UK
}

Received: 9 December 2003 - Revised: 31 August 2004 - Accepted: 9 September 2004 - Published: 22 December 2004

\begin{abstract}
Multi-spacecraft space observations, such as those of CLUSTER, can be used to infer information about local plasma structures by exploiting the timing differences between subsequent encounters of these structures by individual satellites. We introduce a novel wavelet-based technique, the Local Wavelet Correlation (LWC), which allows one to match the corresponding signatures of large-scale structures in the data from multiple spacecraft and determine the relative time shifts between the crossings. The LWC is especially suitable for analysis of strongly non-stationary time series, where it enables one to estimate the time lags in a more robust and systematic way than ordinary cross-correlation techniques. The technique, together with its properties and some examples of its application to timing analysis of bow shock and magnetopause crossing observed by CLUSTER, are presented. We also compare the performance and reliability of the technique with classical discontinuity analysis methods.
\end{abstract}

Key words. Radio science (signal processing) - Space plasma physics (discontinuities; instruments and techniques)

\section{Introduction}

Consider a fleet of spacecraft that is crossing a plasma discontinuity (e.g. a shock front, a large-scale structure), yielding a series of measurements that show resembling patterns with different crossing times. The correct identification of these patterns and the resulting crossing times are key ingredients for determining the orientation and the velocity of the discontinuity. This timing problem has received considerable attention in the analysis of multi-point measurements from the four CLUSTER (Escoubet et al., 1997) spacecraft.

Usual solutions to this timing problem involve direct visualization or, more exceptionally, correlation analysis between the different signals or explicit modeling (Paschmann and Daly, 1998; Haaland et al., 2004). The simplicity of these

Correspondence to: J. Soucek

(soucek@ufa.cas.cz) approaches often stands out against the sophistication of the techniques used for later analysis, such as the inference of the spatial geometry of the plasma structures (Mottez and Chanteur, 1994). Timing problems are easy to handle when the same sharp discontinuity is observed by all satellites. This is sometimes the case with quasi-perpendicular shock crossings. Unfortunately, the situation quickly degrades as soon as the four spacecraft do not see the same structures, or if the discontinuity has a finite extension.

Our objective is to show that the concept of local correlation (Bendat and Piersol, 2000) can be successfully applied in such cases and is much more appropriate than classical correlation techniques that require a certain degree of stationarity of the data. We introduce a local wavelet correlation (LWC) technique suitable for correlating non-stationary time series, such as those obtained from space plasma experiments. This multiscale method, based on the continuous wavelet transform (CWT), allows for a more flexible identification of discontinuities, offering both higher resolution and better robustness. After a description of the method in Sect. 2, and of its properties in Sects. 3 and 4, we shall focus on a series of examples taken from CLUSTER data (Sect. 5) and make comparisons with other approaches.

\section{The local wavelet correlation: description of the method}

The timing problem described above is often solved using an ordinary time domain cross-correlation by choosing a section of each time series containing the structure of interest and shifting those two signals in time with respect to each other, until the cross-correlation of the shifted signals is maximized. The time shift maximizing the correlation is then used as an estimate of the time lag between the observations of the same structure by the two satellites. This technique, however, requires that the time lag does not change within the correlation window. Since the time series under consideration are strongly non-stationary, this assumption is not 
satisfied and the result depends significantly on the choice of this window. The local correlation technique described below is much better suited to analysis of non-stationary data and eliminates this major drawback of the traditional method.

The concept of local correlation function (LCF) as a tool for correlating two time series $f(t)$ and $g(t)$ locally in the neighborhood of two fixed points $\left(t_{1}\right.$ for $f(t)$ and $t_{2}$ for $g(t)$ ) is well known from the classical literature on signal processing (Bendat and Piersol, 2000; Boashash, 1992). This local approach is perfectly appropriate for the timing analysis of strongly non-stationary data sets, like those shown in this article, where the correlation varies significantly over time. Due to the limited statistical content of the data, the question of the choice of the proper estimator of the local correlation is critical to the analysis.

In Perrin et al. (1999) the authors introduced a waveletbased estimator of the local correlation and they applied it successfully to match stereoscopic images. A similar wavelet technique was described previously in Kawata and Arimoto (1996). In this article we introduce a similar wavelet based estimator of LCF, the local wavelet correlation (LWC), which is more appropriate for timing analysis of the time series from multiple spacecraft.

To estimate the LCF, we perform a multiscale decomposition using the Continuous Wavelet Transform (CWT), decomposing the time series into a set of components that contain information about the data at different scales. For each time step, we obtain a set of wavelet coefficients that uniquely describe the signal and its local properties at that time, such as the signal level, the slope, the smoothness, etc. If a similar pattern or structure appears at two different times, then the wavelet coefficients at these times of occurrence should also match. The degree of matching thereby provides a measure of similarity between the two structures. At this point, no assumptions are made on what may cause dissimilarity (shock acceleration, spatial structures, ...). The procedure thus consists in taking a pair of records, comparing their wavelet coefficients pairwise and looking for possible coincidences. Structures that undergo modifications can still be recognized (Perrin et al., 1999), making the LWC really a pattern matching tool.

The LWC is a multiscale method in the sense that it operates simultaneously on different time scales (in contrast to the cross-correlation function, where the dominant scale is set by the length of the window). Indeed, the method automatically adapts itself to the dominant scales of the record: it focuses on small scales if the signal locally contains smallscale structures only, and on large scales otherwise. Several studies have shown that the human eye essentially proceeds in the same way, always trying to extract the most relevant scales (Marr, 1982). Owing to this, the method can achieve a much better time resolution than classical correlation approaches, going as far as the sampling period. Furthermore, by properly choosing the wavelet, one can automatically get rid of trends and offsets. All these properties make the method much more amenable to a systematic analysis of multi-satellite data.
Let $f(t)$ and $g(t)$ represent two time series to be compared and let their CWT be defined as (Daubechies, 1991; Mallat, 1998):

$\mathcal{W}[f](a, \tau)=\int_{-\infty}^{+\infty} f(t) \psi_{a, \tau}^{*}(t) d t$,

where $\psi_{a, \tau}(t)$ is the base wavelet derived by shifting and rescaling a chosen mother wavelet function $\psi$ and $*$ denotes complex conjugation.

$\psi_{a, \tau}(t)=\frac{1}{\sqrt{a}} \psi\left(\frac{t-\tau}{a}\right)$.

The CWT can be understood as a generalization of the windowed Fourier transform in the sense that it yields the spectral decomposition of the signal as a function of time. The concept of spectral decomposition is more general in this context where various shapes of base functions may be used, but the wavelet scale $a$ still roughly corresponds to the inverse value of frequency $1 / f$, since it specifies the width of the base function. The most important advantage of the wavelet transform is its optimal trade-off between time resolution and frequency resolution. Since the mother wavelet function $\psi_{a, \tau}(t)$ is localized in time and its effective width is given by its scale $a$, each wavelet coefficient $\mathcal{W}[f](a, \tau)$ carries information about the input signal $f$ in a neighborhood of time $\tau$, with the width of this neighborhood being proportional to the scale $a$. As the width of the neighborhood decreases with decreasing scale (increasing frequency), the time resolution increases, yielding the above mentioned relation between spectral and temporal resolution.

In our study we shall use real wavelets, but the expressions remain valid for complex signals. Let us first normalize the wavelet coefficients at each time step $\tau$, in order to remove any dependence of the LCF on the local signal power:

$\overline{\mathcal{W}}[f](a, \tau)=\mathcal{W}[f](a, \tau) / \int_{0}^{\infty}|\mathcal{W}[f](\alpha, \tau)|^{2} \frac{d \alpha}{\alpha}$.

We then compute the cross-correlation between the normalized wavelet transforms of $f(t)$ and $g(t)$, as measured, respectively, at times $t_{1}$ and $t_{2}$. The definition of the LWC simply reads

$\gamma\left(t_{1}, t_{2}\right)=\int_{0}^{\infty} \overline{\mathcal{W}}[f]\left(a, t_{1}\right) \overline{\mathcal{W}}^{*}[g]\left(a, t_{2}\right) \frac{d a}{a}$.

Values of the LWC range between -1 and 1 and can thus be interpreted as a measure of similarity between the function $f(t)$ in a neighborhood of the point $t_{1}$ and function $g(t)$ in a neighborhood of $t_{2}$. Given a pattern that is observed in $f$ around time $t_{1}$, we can say that it matches a similar pattern observed in $g$ around time $t_{2}$ if $\gamma\left(t_{1}, t_{2}\right)$ exceeds a given threshold. The difference $\delta t=t_{2}-t_{1}$ then corresponds to the time lag of interest.

The above definition is appropriate for the matching of any type of time series; for our purposes, it is often desirable to make the results more robust, at the expense of the time resolution, as we know that the time lag should not vary abruptly 
in time. To do so, we slightly modify the definition of the LWC by averaging the integrand in Eq. (4) over a short time interval. This averaging first yields

$$
\begin{aligned}
& s\left(t_{1}, t_{2}\right)=\int_{0}^{\infty} \frac{d a}{a} \\
& \int_{-\Delta \tau}^{\Delta \tau} \overline{\mathcal{W}}[f]\left(a, t_{1}+\tau\right) \overline{\mathcal{W}}^{*}[g]\left(a, t_{2}+\tau\right) u(a, \tau) d \tau,
\end{aligned}
$$

where the averaging is carried out over a two-dimensional window $u(a, \tau)$; the constant $\Delta \tau$ defines the maximum width of this window. Finally, the LWC is obtained by normalizing the local correlation between -1 and 1

$$
\begin{aligned}
& \gamma\left(t_{1}, t_{2}\right)=s\left(t_{1}, t_{2}\right) / \\
& \sqrt{\iint\left|\overline{\mathcal{W}}[f]\left(a, t_{1}+\tau\right)\right|^{2} u(a, \tau) \frac{d a d \tau}{a} \cdot \iint\left|\overline{\mathcal{W}}[g]\left(a, t_{2}+\tau\right)\right|^{2} u(a, \tau) \frac{d a d \tau}{a}} .
\end{aligned}
$$

The window $u(a, \tau)$ should be designed consistently with the properties of the wavelet transform: its width in time should be proportional to the wavelet scale in order to keep the time-frequency resolution trade-off provided by the CWT. For example, such a window can be built from an arbitrary one-dimensional window function $w(t)$ (rectangular, triangular, Gaussian, etc.) by rescaling it proportionally to the wavelet scale $a$ and normalizing the window so that the integral $\int u(a, \tau) d \tau$ remains independent of $a$ :

$u(a, t)=\frac{1}{a} w(t / a)$.

Using this window we achieve the goal of adapting the window size to the wavelet scale but keeping equal weights for each scale.

The LWC can be tuned in several ways to the properties of a data set. The main parameters are: the choice of mother wavelet function $\psi$, the width and shape of the averaging window (given by $\Delta \tau$ and the function $w(t)$ in Eq. 7), and the upper and lower bounds $a_{\min }$ and $a_{\max }$ of the range of scales over which the wavelet coefficients are integrated.

In real world applications, one always deals with finitelength discretely sampled signals and a discrete form of the above expressions, in which integrals are replaced by sums. The resolution of the data already sets some limitations on the choice of the above parameters. A minimum value of $a_{\text {min }}$ is determined by the sampling frequency to be approximately equal to $2 T_{\text {samp }}$, where $T_{\text {samp }}$ is the sampling period. One may occasionally want to take a larger value of $a_{\min }$ to discard small scales, which are easily affected by noise.

The maximum scale $a_{\text {max }}$ should be significantly smaller than the size of the data set, to reduce the impact of the boundary errors of the CWT. Such boundary effects, however, can be significantly reduced by using wavelets that have a sufficiently large number of vanishing moments $(N>4)$. With such wavelets, the CWT is invariant with respect to polynomial trends of the order of $N-1$ (Mallat, 1998). We may therefore detrend the original data with a 4th order polynomial (forcing its value and its first derivative at the end points to vanish), to eliminate boundary effects without losing any pertinent information. Finally, as is usual with the CWT, the scales $a$ should be logarithmically spaced. A good compromise between redundancy and computational burden is provided by the array $a=\left[a_{\min }, \sqrt{2} a_{\min }, 2 a_{\min }, \ldots, a_{\max }\right]$.

\section{Application to timing analysis to satellite data}

Before analyzing the timing of various CLUSTER data sets, let us first consider a test case that illustrates the properties and the potential pitfalls of the method. The data set consists of local electron plasma frequency measurements made by the WHISPER instrument (Décréau et al., 1997) on board the CLUSTER spacecraft during multiple magnetopause crossings - see the top panel of Fig. 1. Since the closely separated spacecraft are crossing the same discontinuities, both signals exhibit similar structures with a mere shift in time. Our final objective will be the accurate assessment of this time shift.

A visual inspection of the time series in Fig. 1 already suggests that the time lag continuously evolves in time (even changing sign). Note also that the two spacecraft rarely see the same type of structure, which makes the timing analysis quite a challenge here.

We estimate the time lag as follows: first the LWC is computed from the CWT using Eqs. (3), (5) and (6). The function $f(t)$ denotes the record from spacecraft $\mathrm{C} 1$ and $g(t)$ is from C2. Next, we compute the local correlation $\gamma(t, t+\delta t)$ for a range of lags $-\Delta T<\delta t<\Delta T$ (where $\Delta T$ is an upper bound set by the user) and for all the samples of the record. The results are stored in a matrix which is displayed in the bottom panel of Fig. 1. For each time step, we now determine the lag $\delta t_{\max }$ that maximizes the local correlation. This will be our estimate of the timing difference; see the middle panel of Fig. 1. Note that the values of $\delta t_{\max }$ are not restricted to integer multiples of the sampling period, as better resolution may be achieved by locally fitting a parabola to the maximum of the LWC. In this example, the reference signal is from spacecraft $\mathrm{C} 1$, so the results can be interpreted as the time it takes for patterns observed by $\mathrm{C} 1$ at time $t$ to reappear at $\mathrm{C} 2$.

Before interpreting these results, let us comment on how the LWC analysis proceeds. As specified before, the LWC is data-adaptive in the sense that it automatically selects the range of scales in which the power content is the largest. At the magnetopause crossing of 06:05:20 UT, the dominant pattern is the step-like discontinuity, which affects all scales alike. The LWC clearly reaches a single and well-defined maximum with a peak correlation of 0.3 . The value of correlation may seem rather low here, but note that the signals are significantly dissimilar in the neighborhood of the discontinuity (a decrease in density behind the crossing is seen only at C1). Regardless of this dissimilarity, the LWC identified the correct time lag between the fronts of the MP crossings. 

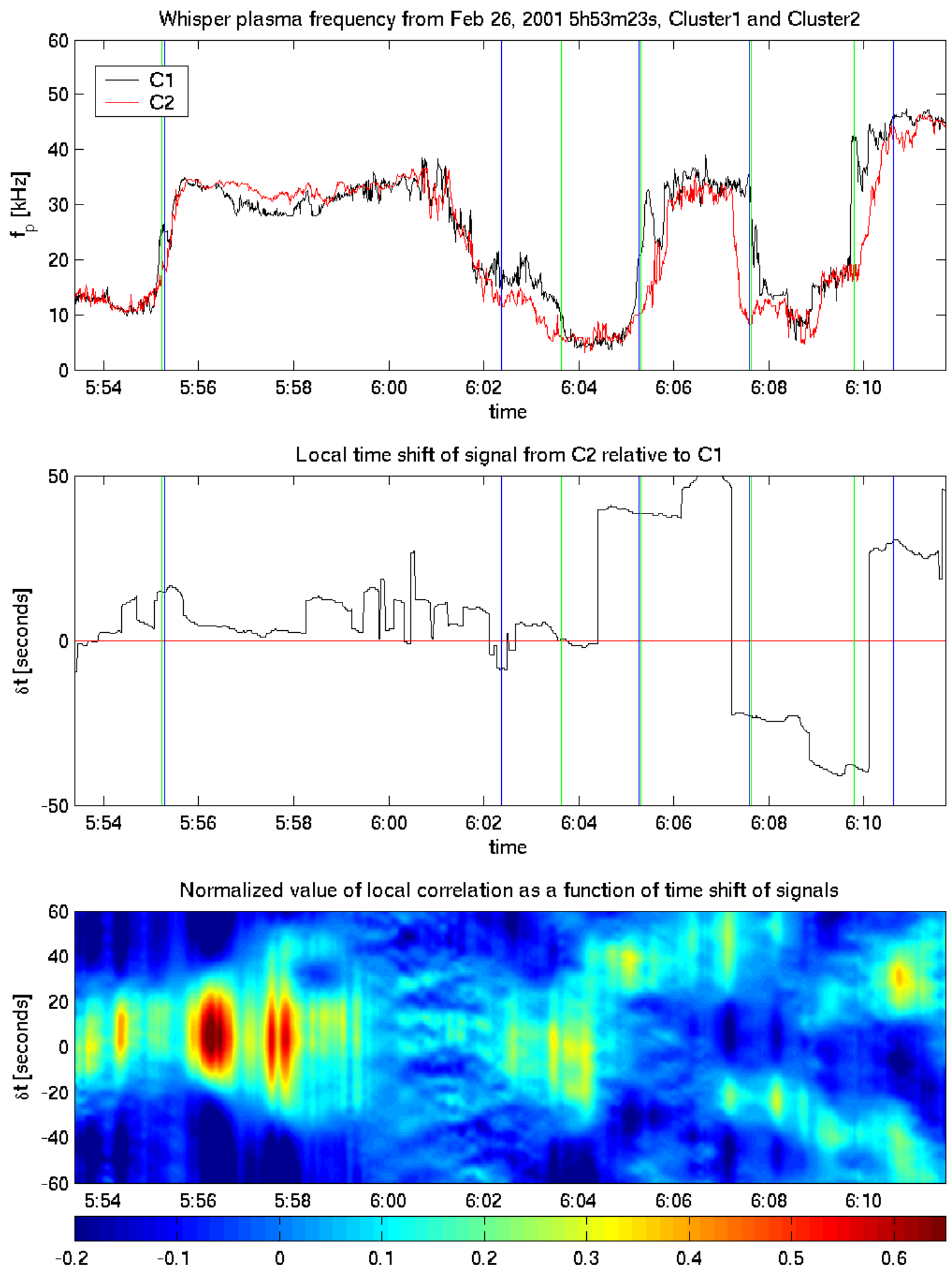

Fig. 1. Example of LWC timing, applied to the electron density measured by WHISPER during repetitive magnetopause crossings on 26 February 2001, from 5:54-6:11 UT. The upper panel shows the plasma frequency as measured by spacecraft C1 and C2. The middle panel provides the time lag for which the LWC is maximum. Positive values imply that $\mathrm{C} 1$ is preceding $\mathrm{C} 2$. The bottom panel displays the LWC versus lag and time; the color scale ranges from -0.2 to 0.65 . The approximate location of five magnetopause crossings are indicated by vertical green lines. In this example, 10th order Daubechies wavelets were used with a window derived from a 1-D Gaussian window. 
A similar situation, where a correct time shift is identified correctly by a single sharp maximum of the LCF, occurs for the next crossing (06:08 UT).

A different situation occurs at 6:00 UT, where the LWC tries to lock on small scales associated with weak wave activity. The small amplitude of these fluctuations is not problematic per se, since the wavelet coefficients are normalized versus local power. The main problem comes from the lack of similarity between the two records. Not surprisingly, the absolute value of local correlation remains rather low (typically -0.1 to 0.1 ). It also exhibits multiple maxima and the estimated lag varies erratically. The concept of LCF is inapplicable here.

\section{Pitfalls and validation of the results}

The preceding example reveals several potential pitfalls, whose understanding is the key to a proper interpretation of the LCF analysis results.

In the first place, we must stress that a time lag can be properly and unambiguously defined only for signals that are identical up to a shift in time. In the more realistic situation, when the signals are similar but not identical, pattern matching is required. A threshold must be selected below which the patterns are too dissimilar to justify the definition of a time lag. This occurs between 05:57 and 06:00 UT in Fig. 1.

A different problem is raised by the magnetopause crossing at 05:55 UT. Spacecraft $\mathrm{C} 1$ observes in the middle of the ramp a small dip that goes unseen by $\mathrm{C} 2$. The LWC algorithm correctly matches the ramp of $\mathrm{C} 2$ with the first part of C1's ramp, yielding a time lag of approximately $10 \mathrm{sec}-$ onds. However, it fails to resolve the second part of the ramp, where the two signals almost coincide.

These effects are a direct consequence of the properties of the CWT. Each wavelet coefficient $\mathcal{W}(a, t)$ contains information about the input signal in a neighborhood of the point $t$. The width of this neighborhood is given by the wavelet width which is proportional to the scale. Discontinuities or abrupt changes in the signal contribute to wavelet coefficients at a wide range of scales, analogously to a Fourier decomposition of a step function. The localization of this contribution in time is again given by the scale of the wavelet. In Fig. 2 the spiky structures corresponding to such discontinuities in the signal can be clearly recognized.

When the LCF is estimated using Eq. (5), we match 2-D arrays of wavelet coefficients. Obviously, the spiky structures discussed above contribute very significantly to the result, since they cover a wide range of scales. The presence of such structures may influence the wavelet coefficients at large scales (and consequently the LWC), considerably far from the discontinuity. Now assume that a relatively smooth structure appears in the signal at a time $t$, close to a sharp discontinuity at a time $t^{\prime}$. The contribution of the nearby discontinuity at $t^{\prime}$ to the wavelet transform at $t$ may actually be stronger than that of the smoother structure present at time $t$. Consequently, the LWC at the time $t$ may reflect the correlation of stronger nearby structures. These different effects explain the behaviour of the LWC at the magnetopause crossing of 5:55 UT. They also explain the unusually high level of correlation that appears just after the magnetopause crossing, from 05:56 to about 05:58 UT. The signals here are barely correlated, but since their variance is low most wavelet coefficients are contaminated by the presence of the nearby magnetopause discontinuity.

Another consequence of this contamination is the appearance of multiple maxima of comparable magnitude in LCF. An example of this multi-modality appears in the magnetopause crossing at 06:10 UT (see Fig. 1). The correlation matrix (bottom panel) exhibits two ridges: one in the region of positive shifts, which corresponds to the true 06:10 UT crossing, and another ridge that is simply a consequence of the overlap from the previous magnetopause crossing. As one moves further away from the crossing, the overlap correlation becomes weaker and finally, the correct local maximum gets selected. Again, the problem does not really come from the method itself, but from the comparison of two structures that are dissimilar.

From the examples discussed above, it becomes evident that the peak value of the correlation is not a sufficient indication of the reliability of the time shift estimate. Specifically, a high level of correlation may not correspond to a correct estimate, since multiple possibilities of pattern matching often exist.

Most of the discussed effects can be significantly reduced by feeding physical information into the algorithm, such as the range of scales over which the LWC is integrated. Beforehand, we need some independent criterion to assess the validity of the results. Two such criteria are proposed below.

\subsection{First validation criterion: multi-wavelet statistics}

The first validation criterion is based on the idea that the estimated time shift should depend on the input signals only, and not on the wavelets ${ }^{1}$. Tests carried out on synthetic signals indeed do not show significant differences between various families of wavelets, with orders ranging from 4 to 20 . Some noticeable exceptions are the Haar, the Morlet and the Gaussian hat wavelets, whose performance is systematically lower.

We built a statistical ensemble by computing the time shifts $\delta t$ from a set of different wavelet functions and determined the distribution function (histogram) of the multiple $\delta t$ for each time $t$. Such a histogram is shown in the bottom panel of Fig. 3. For strongly correlated structures, the time lags are independent of the mother wavelet and so the his-

\footnotetext{
${ }^{1}$ This is not exactly true: different families of wavelets (Daubechies, symlets, Haar, Morlet, ... ) will not capture the features of the data in the same way. The order of the wavelets also affects the outcome. Low order wavelets (say, $N<4$ ) are more suitable for irregular signals, whereas large order wavelets offer a better resilience against trends.
} 

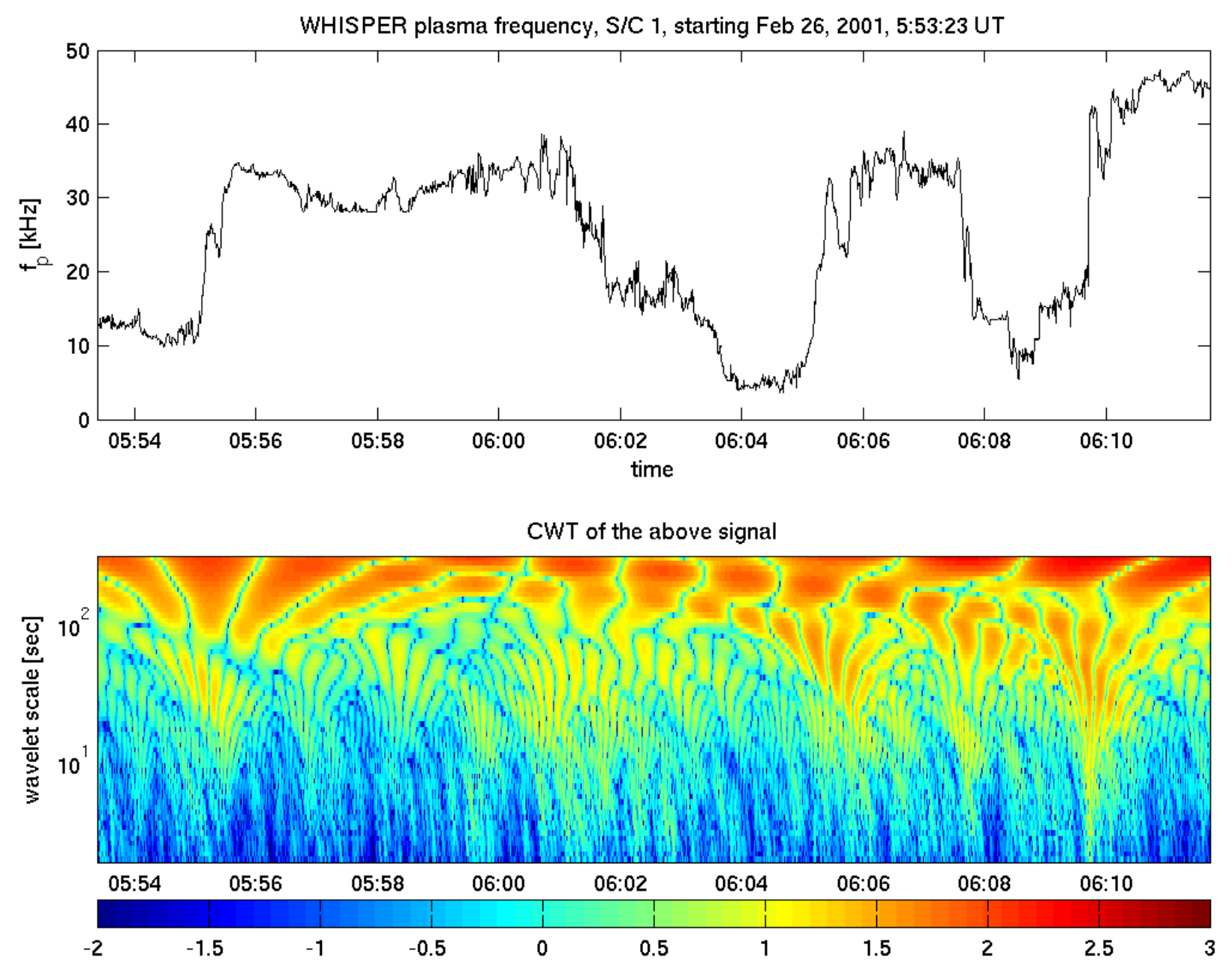

Fig. 2. The continuous wavelet transform of the density data of spacecraft $\mathrm{C} 1$ from Fig. 1 . The lower panel shows the logarithm of $\mathcal{W}(a, \tau)$. The CWT was computed using 10th order Daubechies wavelets.

togram should exhibit a single narrow peak. The width of this peak can be quantified by its standard deviation:

$s_{\mathrm{mw}}(t)=\sqrt{\sum_{\text {wavelets }}\left(\delta t-\delta t_{\max }\right)^{2} p(t, \delta t)}$,

where $p(t, \delta t)$ is the empirical probability of observing a lag $\delta t$ at time $t$ (estimated from a statistical ensemble described above) and $\delta t_{\max }$ is defined as in Sect. 3.

A necessary (but not sufficient) condition for the reliability of the time lag estimates is obtained by comparing the standard deviation $s_{\mathrm{mw}}$ with a given threshold. Every time the value of $s_{\mathrm{mw}}$ exceeds this threshold the estimated time lags should be considered invalid. Figure 3 indeed shows that this criterion flags out the ambiguous magnetopause crossings at 06:05, 06:08 and 06:10 UT. With this criterion, however, the ambiguous magnetopause crossing between 06:02 and 06:04 is not rejected. That particular crossing does not suffer from multimodality, so an additional criterion is needed.

\subsection{Second validation criterion: triangular differences}

The second validation criterion is more specific to the CLUSTER experiment with its four spacecraft arranged in a tetrahedral configuration; it may also be adapted to other configurations. Given the four satellites, the time shifts can be estimated from 6 satellite pairs. Let $\Delta t_{i j}$ denote the time shift from the signal of spacecraft $i$ with respect to the signal of spacecraft $j$. Out of the 6 time shifts, only 3 are independent; their linear dependence can be expressed by the following set of equations:

$$
\begin{aligned}
& \Delta t_{21}-\Delta t_{31}+\Delta t_{32}=0 \\
& \Delta t_{31}-\Delta t_{41}+\Delta t_{43}=0 \\
& \Delta t_{42}-\Delta t_{41}+\Delta t_{21}=0 \\
& \Delta t_{32}-\Delta t_{42}+\Delta t_{43}=0 .
\end{aligned}
$$

Geometrically, the above relations express the conservation of the oriented sum of the time shifts along the edges of each of the four sides of the tetrahedron, hence the name "triangular differences". 

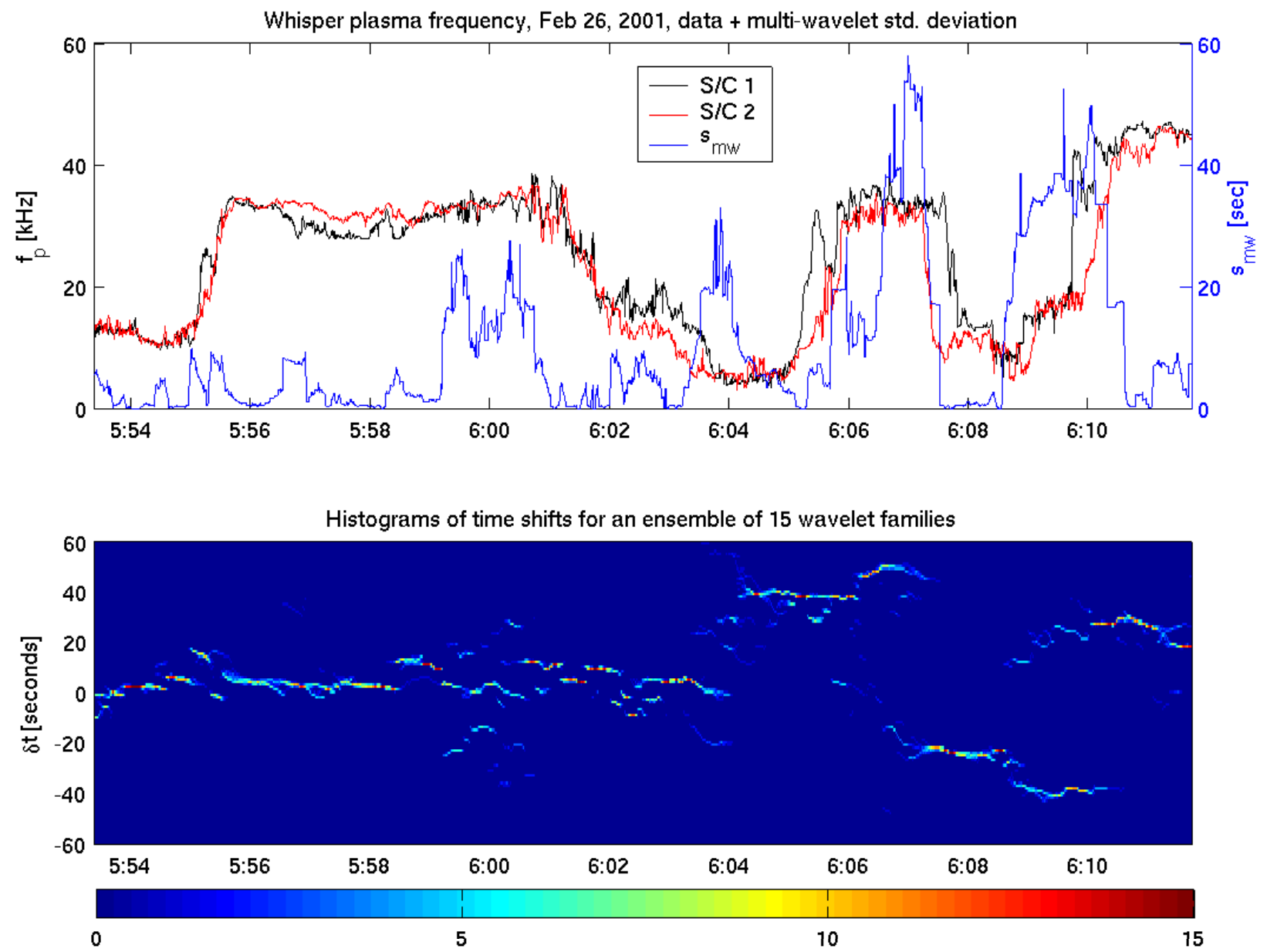

Fig. 3. The multi-wavelet criterion as applied to the density data of Fig. 1. The upper panel displays the density measurements, together with the standard deviation $s_{\mathrm{mw}}$. The bottom panel displays the histogram of the time shifts estimated using an ensemble of 15 different mother wavelets.

The application of the above relations to the validation problem is immediate: we compute all four left-hand sides of Eqs. (9-12) and compare them with a selected threshold. Note, however, that the time shifts are computed with respect to different reference signals. In Eq. (9), for example, the values $\Delta t_{21}$ and $\Delta t_{31}$ evaluated at time $t$ give the time shift relative to a structure appearing in signal 1 at the time $t$. However, the same structure appears at time $t+\Delta t_{21}$ in the signal 2, and so the corrected form of Eq. (9) should actually read:

$T D_{1}=\Delta t_{21}(t)-\Delta t_{31}(t)+\Delta t_{32}\left(t+\Delta t_{21}\right)=0$.

In Fig. 4 we plot the four triangular differences $T D_{i}$ (bottom panel), as well as their sum $T D_{\mathrm{all}}=\sum_{i=1}^{4}\left|T D_{i}\right|$ (middle panel - black line). The smaller this quantity, the more consistent the lags are. One can check that all 6 time shifts are consistent for the magnetopause crossings at 05:55, 06:05 and 06:08 UT. The ambiguous crossing around 06:03 is now correctly identified.

When analyzing plasma discontinuities, it is desirable to know both the lag and the time of occurrence of the discontinuity. For sharp discontinuities, this is straightforward, as one simply takes the lag $\delta t\left(t_{\mathrm{D}}\right)$ at the time when the discontinuity is strongest in the reference signal. If, however, the discontinuity has a finite width, then the validation criteria can help by letting us take instead the time near the discontinuity when the lags are most reliable. These times are marked by the blue vertical lines in Fig. 4. Comparing them with the green lines obtained by visualization, we can check that the estimates are indeed consistent.

\section{Estimating normal velocity vectors of discontinuities}

We finally consider a series of examples where the LWC timing is used to estimate the orientation and normal velocity of the Earth's bow shock and the magnetopause. These results are compared to those obtained by standard methods (coplanarity and minimum variance techniques). All examples are based on the data collected by the four CLUSTER satellites when the latter were in an approximately tetrahedral configuration, with a spacecraft separation of about $600 \mathrm{~km}$. 

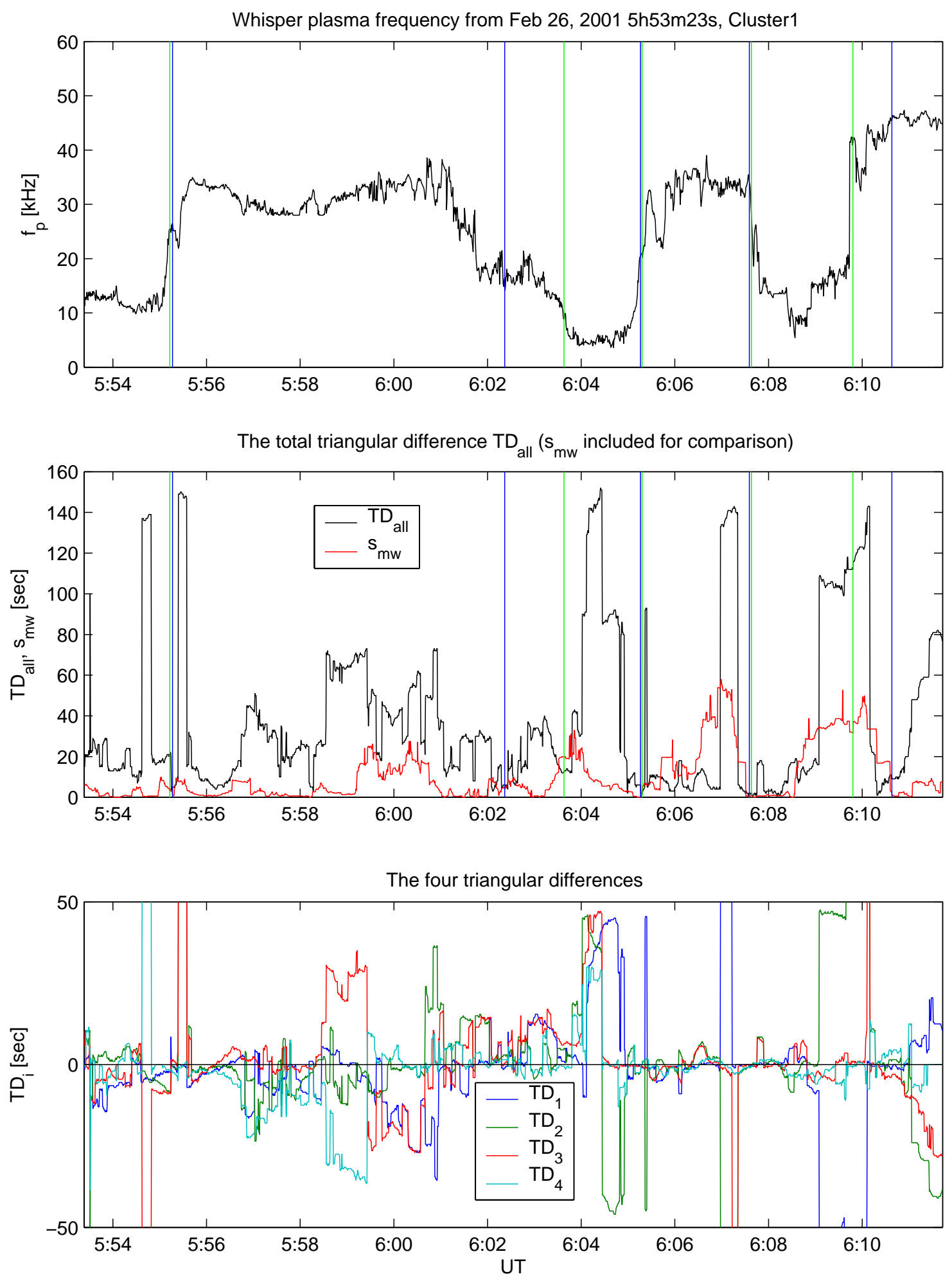

Fig. 4. The triangular differences between the time lags, as computed between all six satellite pairs. The data are the same as in Fig. 1. The upper panel shows the density data from spacecraft $\mathrm{C} 1$ only. The bottom panel displays all four triangular differences. The sum $T D_{\text {all }}$ of the triangular differences, and the standard deviation $s_{\mathrm{mw}}$ are displayed in the central panel. 


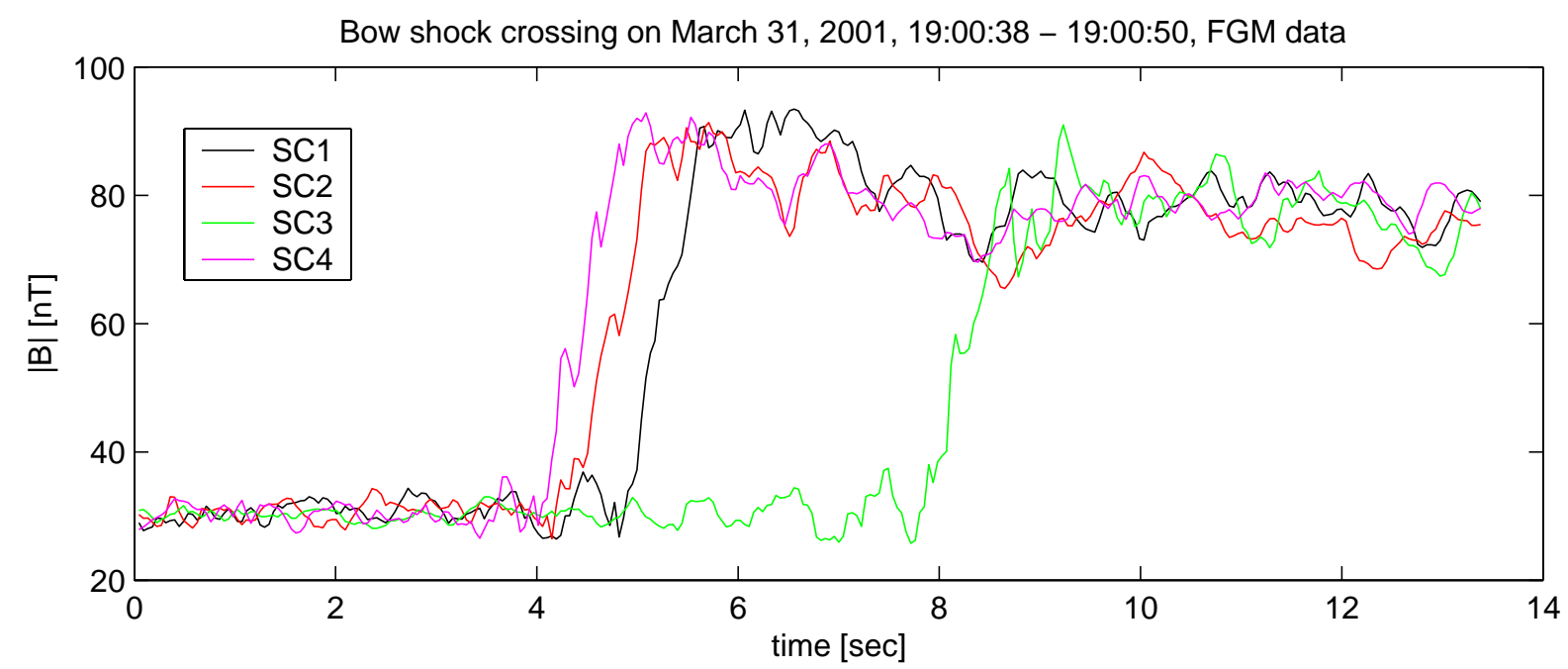

The above dataset, time-aligned using LCF timing

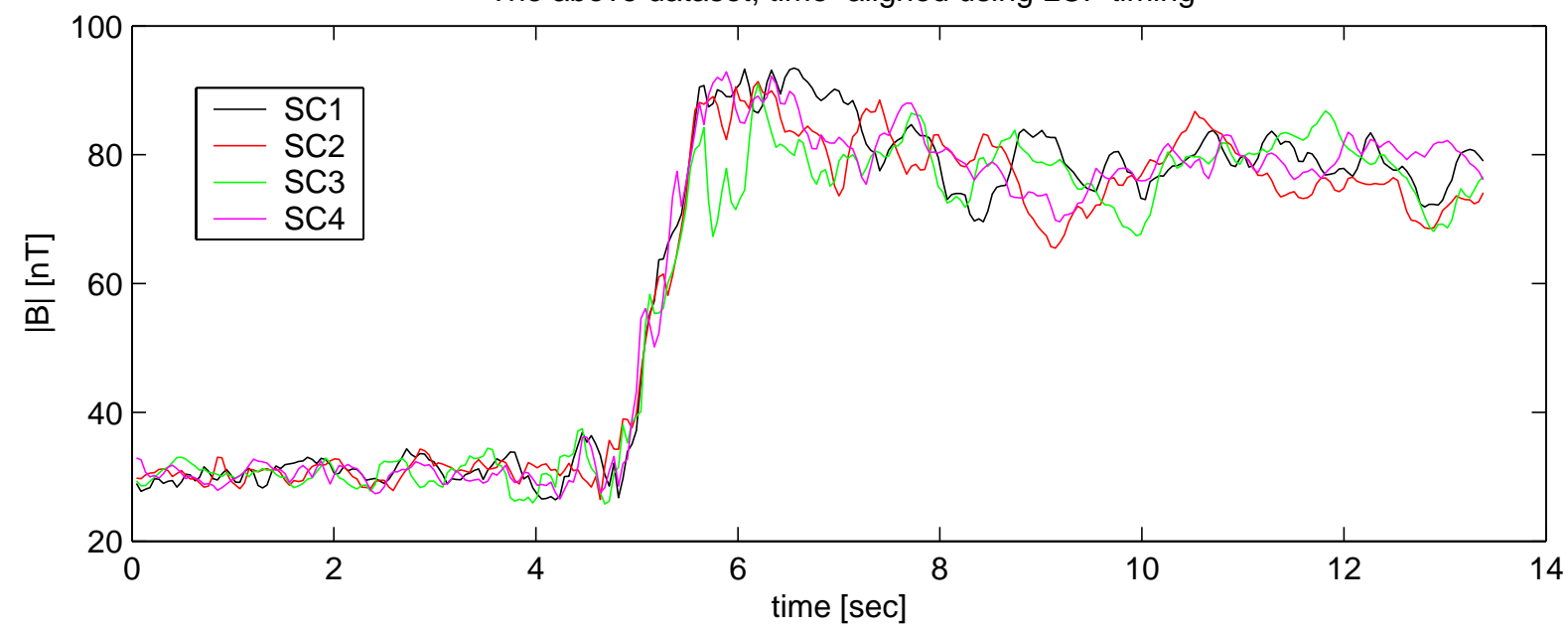

Fig. 5. A LWC analysis of a sharp, quasi-perpendicular bow shock crossing of 31 March 2001, from 19:00:38-19:00:50 UT. The upper panel shows a magnetic field magnitude observed by the FGM instrument on CLUSTER during the bow shock crossing. The bottom panel shows the same data but with the shock fronts shifted by the lags obtained from the LWC. The sampling frequency of the magnetic field data is $22.5 \mathrm{~Hz}$.

The normal velocity vector is inferred from the timing information using the classical method (Paschmann and Daly, 1998; Dunlop et al., 2001): let $\Delta t_{i j}$ denote the time shift estimated between signals from satellites $i$ and $j$, as defined in Sect. 4.2. Let also $\boldsymbol{r}_{i}$ denote the position of spacecraft $i$. Assuming that the crossed boundary or structure is planar and moving with uniform velocity, we can now estimate its unit normal vector $\boldsymbol{n}$ and magnitude of normal velocity $|\boldsymbol{V}|$ by solving a simple set of linear equations:

$\frac{1}{|\boldsymbol{V}|}\left(\boldsymbol{r}_{i}-\boldsymbol{r}_{j}\right) \cdot \boldsymbol{n}=\Delta t_{i j}(t), \quad i, j=1 \ldots 4, \quad i<j$.

In the case of four satellites this results in an overdetermined set of 6 linear equations with 3 unknowns, which can be solved using standard least-squares techniques.

The above method relies on the rather strong assumptions of planarity and uniform velocity. Recent studies based on
CLUSTER data (Horbury et al., 2002) suggest that in the case of terrestrial bow shock, and for a satellite separation of several hundred kilometers, the above assumptions are usually satisfied with reasonable accuracy. On the other hand, the magnetopause can still be approximated by a planar structure, but its acceleration is often significant enough to invalidate the uniform velocity approximation (Dunlop et al., 2001).

\subsection{First example: quasi-perpendicular shock crossing}

Figure 5 illustrates an application of the LWC timing to magnetic field measurements obtained by the FGM magnetometer (Balogh et al., 1997) during a quasi-perpendicular bow shock crossing. The shock ramp is relatively steep and the profile is very similar on all spacecraft. Not surprisingly, the timing is well defined and no fine tuning is necessary. We 

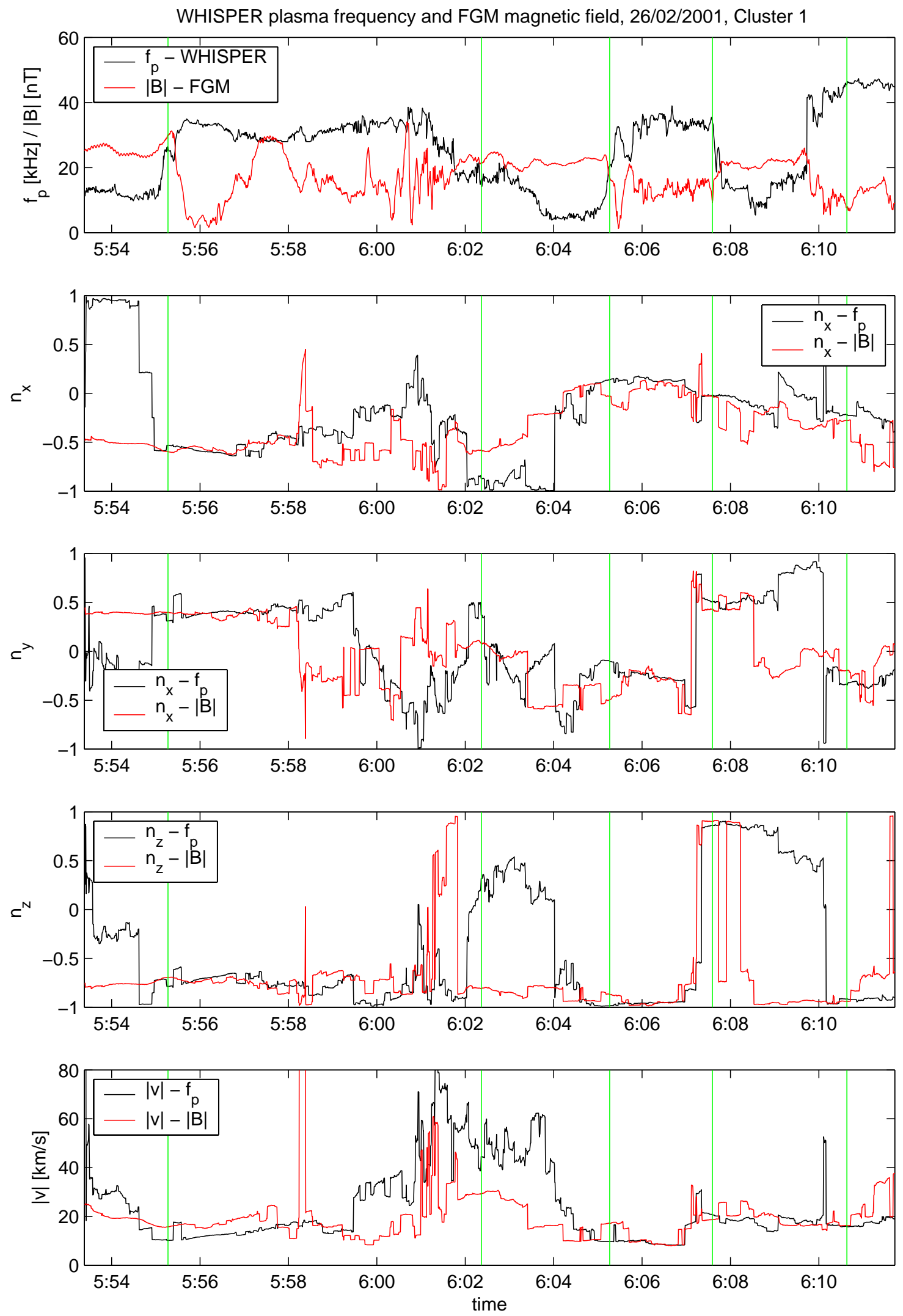

Fig. 6. Application of the LWC to multiple magnetopause crossings on 26 February 2001, from 05:53:30-06:11:30 UT. The upper panel shows the input data from spacecraft $\mathrm{C}$ 1: total magnetic field from FGM (resampled at $4 \mathrm{~Hz}$ ), and plasma frequency from WHISPER (sampled at $2 \mathrm{~Hz}$ ). Panels 2 to 4 display the three components of the normal vector $n$ of the discontinuities as determined from the magnetic field and the electron density data. The bottom panel shows the magnitude of the velocity. 
Table 1. Normals from various methods.

\begin{tabular}{llllcc}
\hline Time & LWC normal & Timing normal & MV normal & Angle MV-LWC & Angle Timing-LWC \\
\hline $05: 56$ & {$[-0.50,0.22,-0.84]$} & {$[-0.37,0.13,-0.92]$} & {$[0.24,-0.08,-0.97]$} & $47.6^{\circ}$ & $10.2^{\circ}$ \\
$06: 02$ & {$[0.76,-0.30,0.58]$} & {$[-0.05,-0.15,0.99]$} & {$[0.36,-0.11,0.93]$} & $32.7^{\circ}$ & $54.6^{\circ}$ \\
$06: 08$ & {$[-0.07,-0.34,-0.94]$} & {$[0.01,-0.39,-0.92]$} & {$[0.25,-0.26,-0.93]$} & $18.9^{\circ}$ & $5.5^{\circ}$ \\
$06: 10$ & {$[0.21,-0.05,0.98]$} & {$[0.20,0.06,0.98]$} & {$[0.45,0.07,0.89]$} & $16.2^{\circ}$ & $6.3^{\circ}$ \\
\hline
\end{tabular}

used 10th order Daubechies wavelets. Averaging was performed over 52 logarithmically spaced wavelet scales from $a_{\min }=0.4 \mathrm{~s}$ to $a_{\max }=30 \mathrm{~s}$.

\subsection{Second example: multiple magnetopause crossings}

Let us now return to the multiple magnetopause crossings of 26 February 2001, which served before as a test case. Figure 6 shows the normal components of the the magnetopause crossings (three panels in the middle) estimated using the LCF timing both from FGM magnetic field data and from WHISPER plasma frequency data. As before, green lines indicate the approximate locations of the magnetopause crossings.

Clearly, there is a good agreement between the normal estimates based on the density data and on the magnetic field data for the crossings at 05:55, 06:08 and 06:10 UT. The discrepancies between the results for the other two crossings are a consequence of the problems described above in Sect. 4. Note that the two estimates often significantly differ and tend to agree only in the vicinity of magnetopause crossings, where significant correlated structures are present in all signals. This comparison of multi-instrument data represents an ultimate test of the correctness of the LWC technique.

Minimum variance analysis (Paschmann and Daly, 1998) is a single spacecraft technique for estimating the direction of a discontinuity normal from a magnetic field measurement. This method is completely independent from the interspacecraft timing technique, so we use it as another validation test for the LCF timing-technique. The properties of this classical method are thoroughly studied in the literature (Paschmann and Daly, 1998). Like the timing based techniques, the minimum variance is known to suffer from errors introduced by deviation from the assumptions of planarity and time stationarity of the discontinuity, but the assumption of uniform velocity of the discontinuity is not used in this approach.

Table 1 summarizes magnetopause normals obtained by the two different techniques for the event of 26 February 2001 (see also Fig. 6). The second and third columns of the table contain normals computed from the timing information using Eq. (14). The second column normals use the timing obtained automatically by the LWC and in the third column the timing is estimated by traditional visual comparison and by manual shifting of data sets. The table also shows the normal vector obtained by minimum variance and angles between different normal estimates.

By comparing the LWC timing normals and the normals calculated from timing obtained by visual matching of data, we can evaluate the correctness of the LCF estimates. This comparison confirms the conclusions stated above: for all magnetopause crossings, except for the one at 06:02, the results obtained from the LWC fully agree with the visual timing observations. The relative angles between those normals range form $5^{\circ}$ to $10^{\circ}$. In the case of the 6:02 crossing, the LWC technique fails, as was indicated by the validation criteria in Sect. 4 and the resulting normal is therefore incorrect. Comparing the timing normals with minimum variance normals, we can deduce some information about the acceleration of the magnetopause between the crossings by individual satellites. Following the approach by Dunlop et al. (2001), we assume that the magnetopause is approximately planar but its acceleration on the scale of spacecraft separation may not be negligible. From Table 1, it is evident that for the last two MP crossings the minimum variance and timing normals agree within $20^{\circ}$, so the assumptions of planarity and uniform velocity of the discontinuity are satisfied to a reasonable extent and Eq. (14) is applicable. On the other hand, the first crossing at 05:56 shows a significant deviation of $47.6^{\circ}$ and we can conclude that this is either caused by an acceleration of the magnetopause or by a localized spatial structure.

\section{Summary and conclusions}

In this paper we introduced the Local Wavelet Correlation (LWC) as a novel tool for estimating the timing differences from multi-satellite data. This technique was designed to correlate input signals over a wide range of scales while putting equal weight on each scale. When applied to non-stationary signals, the LWC is more robust than ordinary linear correlation methods, which tend to be biased by large-scale structures of the size of the averaging window. Secondly, the correlation coefficient can be calculated locally, yielding the level of correlation between two time series as a function time, with a very good time resolution.

The LWC was used here to determine the relative time shift between observations of the same plasma structures by 
each of the four CLUSTER satellites. Tests based on a collection of events show that the LWC correctly matches similar patterns and provides consistent time differences whenever these patterns can be unambiguously identified by eye. The method fails when the compared patterns become too dissimilar to justify a matching. We developed for that purpose two independent validation techniques, which allow one to determine the intervals in which the timing results are unreliable.

In the context of multi-point space plasma observations, timing information is essential for determining the orientation and the normal velocity of the bow shock or the magnetopause. Using several examples, we demonstrated that the results obtained by LWC are fully consistent with those obtained by classical methods. One major advantage of the LWC, however, is that it requires no guidance, making it more amenable to a systematic statistical study of large numbers of discontinuity crossings.

Future work includes the possible application of this method to the timing of complex patterns containing substructures of different origin with possibly different velocities. Here we can take advantage of the high time resolution of the LWC and the possibility to focus the correlation on a selected range of scales. A 2-D version of the LWC is presently under development for computing flow velocity fields from SoHO coronagraph images and for doing stereoscopic matching on image pairs from the future Stereo mission.

Acknowledgements. The first author was supported by Program for International Scientific Cooperation (PICS 1175), ESA PRODEX Contract No. 14529 and grant 205/01/1064 of Grant Agency of the Academy of Sciences of the Czech Republic.

Topical Editor T. Pulkkinen thanks two referees for their help in evaluating this paper.

\section{References}

Balogh, A., Dunlop, M. W., Cowley, S. W., Southwood, D. J., et al.: The Cluster magnetic field investigation, Space Sci. Rev., 79, 6592, 1997.

Bendat, J. S. and Piersol, A. G.: Random data, analysis and measurement procedures, Wiley, New York, 2000.

Boashash, B.: Time-frequency signal analysis - methods and applications, John Wiley Press, New York, 1992.

Daubechies, I.: Ten lectures on wavelets, SIAM, 1991.

Décréau, P., Fergeau, P., Krasnoselskikh, V. et al.: WHISPER, a resonance sounder and wave analyser: Performances and perspectives for the Cluster mission, Space Sci. Rev., 79, 157-193, 1997.

Dunlop, M. W., Balogh, A., Cargill, P., et al.: Cluster observes the Earth's magnetopause: coordinated four-point magnetic field measurements, Ann. Geophys., 19, 1449-1460, 2001.

Escoubet, C. P., Russel, C. T., Schmidt, R. et al.: The Cluster and Phoenix Missions, Kluwer Academic Publishers, Dodrecht, 1997.

Haaland, S., Sonnerup, B. U. Ö., Dunlop, M. W., Balogh, A., Hasegawa, H., Klecker, B., Paschmann, G., Lavraud, B., Dandouras, I., and Rème, H.: Four-spacecraft determination of magnetopause orientation, motion and thickness: Comparison with results from single-spacecraft methods, Ann. Geophys., 22, 1347-1365, 2004.

Horbury, T., Cargill, P. J., Lucek, E. A., Eastwood, J., et al.: Four spacecraft measurements of the quasiperpendicular terrestrial bow shock: Orientation and motion, J. Geoph. Res., 107, SSH 10-1, 2002.

Kawata, K. and Arimoto, S.: Signal matching using wavelet correlation, Electronics and Comm. in Japan, 79 (9), 23-34, 1996.

Mallat, S.: A wavelet tour of signal processing, Academic Press, London, 1998.

Marr, D.: Vision: a computational investigation into the human representation and processing of visual information, Freeman, New York, 1982.

Mottez, F. and Chanteur, G.: Surface crossing by a group of satellites: A theoretical study, J. Geoph. Res., 99, 13 499-13 508, 1994.

Paschmann, G. and Daly, P. W.: Analysis methods for multispacecraft data, Kluwer, Amsterdam, 1998.

Perrin, J., Torresani, B., and Fuchs, P.: A localized correlation function for stereoscopic image matching, Traitement du Signal, 16, 3-14, 1999. 